\title{
A predictive model for identifying candidates for adjuvant chemotherapy based on recurrence risk profile of resected, node-negative (NO) non-small cell lung cancer
}

\author{
Timur A. Krivitsky ${ }^{1,2}$, Gavin M. Wright ${ }^{3,4,5}$, Muteb Al Zaidi ${ }^{3,6}$ \\ ${ }^{1}$ Department of General Medicine, St Vincent's Hospital Melbourne, Fitzroy, VIC, Australia; ${ }^{2}$ Department of Medicine, Peninsula Health, \\ Frankston, VIC, Australia; ${ }^{3}$ Department of Cardiothoracic Surgery, St Vincent's Hospital Melbourne, Fitzroy, VIC, Australia; ${ }^{4}$ Department of \\ Surgery, Melbourne University, VIC, Australia; ${ }^{5}$ Victorian Comprehensive Cancer Centre, Melbourne, VIC, Australia; ${ }^{6}$ Division of Thoracic \\ Surgery, King Abdullah Medical City, Makkah, Saudi Arabia \\ Contributions: (I) Conception and design: All authors; (II) Administrative support: All authors; (III) Provision of study materials or patients: GM \\ Wright; (IV) Collection and assembly of data: TA Krivitsky; (V) Data analysis and interpretation: All authors; (VI) Manuscript writing: All authors; \\ (VII) Final approval of manuscript: All authors. \\ Correspondence to: Dr. Timur A. Krivitsky. Department of General Medicine, Peninsula Health, 2 Hastings Rd., Frankston, VIC, 3199 , Australia. \\ Email: timkriva@gmail.com.
}

Background: The decision for administering adjuvant chemotherapy (AC) in completely resected nodenegative non-small cell lung cancer (NSCLC) is guided by likelihood of disease recurrence or death based on tumor, node, metastasis (TNM) stage. However, within each TNM stage are sub-groups of patients that are more or less likely to relapse than stage alone predicts.

Methods: In this retrospective cohort study, prospective data from 394 consecutive patients who underwent complete resection of node-negative NSCLC without adjuvant therapies, between 2002 and 2019 was retrospectively analyzed. Independent tumor and host risk factors for recurrence were subjected to multivariate analysis to develop a predictive risk model distributing patients into low-risk or high-risk categories.

Results: Recurrence risk was independently predicted by a neutrophil:lymphocyte ratio (NLR) of $\geq 3.5$ [hazard ratio (HR), 1.9; 95\% confidence interval (CI), 1.1-3.5], visceral pleural invasion (HR, 2.2; 95\% CI, 1.3-3.8), histopathology other than adenocarcinoma or squamous cell (HR, 2.6; 95\% CI, 1.2-5.5) and tumor size $>33 \mathrm{~mm}$ (HR, 3.9; 95\% CI, 2.3-6.7). The specific combination of risk factors contributed to a score for a risk model which classified $9 \%$ of Stage I and $69 \%$ of Stage $\geq$ II patients as high-risk. The predicted 5 -year disease-free survival (DFS) for high-risk and low-risk patients as scored by the predictive model was $30 \%$ and $85 \%$, respectively.

Conclusions: Our readily reproducible, low-technology model, developed from individually validated tumor/host risk factors, identified sub-groups of resected node-negative NSCLC patients at significantly discordant risk of recurrence to their TNM stage category.

Keywords: Non-small cell lung cancer (NSCLC); recurrence; disease-free survival (DFS)

Submitted Jul 14, 2020. Accepted for publication Nov 06, 2020.

doi: $10.21037 /$ jtd-20-2434

View this article at: http://dx.doi.org/10.21037/jtd-20-2434

^ ORCID: Timur A. Krivitsky, 0000-0003-1614-0617; Gavin M. Wright, 0000-0002-7000-9305. 


\section{Introduction}

Surgical resection is the mainstay of initial treatment for early stage non-small cell lung cancer (NSCLC). Unfortunately, approximately $20 \%$ of patients with resected node-negative NSCLC experience recurrence within two years. At five years, up to one third (1) of such cases may recur. Patients whose tumors recur have a poor prognosis with a three-year overall survival (OS) of around $24 \%$ (2). Adjuvant chemotherapy (AC) is aimed at reducing recurrence and ultimately improves absolute OS for allcomers by $4-5.4 \%$ over five years $(3,4)$. Potential benefit from AC must be weighed up against the potential harms, and therefore the most accurate assessment of recurrence risk is necessary to ensure the correct patients receive it. The therapeutic ratio of $\mathrm{AC}$ would be improved if patients with lowest risk of recurrence could be excluded and those of higher risk be included in the existing treatment algorithm.

Currently, the decision to offer AC for operable NSCLC is informed by guidelines based on large trials and metaanalyses $(4,5)$ using erstwhile TNM staging systems. Due to lack of evidence of overall benefit, and even possible evidence of harm in patients with Stage IA tumors, AC is not routinely recommended. Administration of AC for Stage IB tumors remains controversial and may be considered in cases possessing high-risk features including, poor differentiation, vascular invasion, sub-lobar resection, visceral pleural involvement (VPI), and unknown lymph node status (6). The previous distinction of increased risk with tumors $>4 \mathrm{~cm}$ diameter in IB patients has become largely irrelevant as this tumor diameter has now been upstaged as IIA stage in the latest TNM (eighth revision) system. AC is associated with survival improvement in Stage II and III and is therefore is usually chosen as a treatment strategy in these patients.

It is recognized that there exist sub-groups within all stages of resected NCSLC (I-IIIA) that are more or less likely to experience disease recurrence than their TNM stage alone would suggest. Consequently, a large amount of research has been undertaken in identifying these patients and the specific factors that predict their risk of recurrence. In recent times, many clinical, pathological, and molecular markers have been investigated for their effect on recurrence and survival in NSCLC (7). Although helpful, it is often difficult to translate the conclusions from studies of many of these markers into pragmatic real-world practice for many reasons. These studies are often underpowered, exploring heterogenous populations and/or investigating markers which may not be readily available or affordable.

In essence, the likelihood of cancer relapse relates to the interplay between tumor biology, tumor extent and host response. Using this principle, we aimed to develop a model for predicting recurrence based on readily available information with a view to refining the stratification of recurrence risk in completely resected node-negative NSCLC patients, thereby facilitating a more targeted AC strategy.

We present the following article in accordance with the Transparent Reporting of a Multivariable Prediction Model for Individual Prognosis or Diagnosis (TRIPOD) reporting checklist (8) (available at http://dx.doi.org/10.21037/jtd-202434).

\section{Methods}

The study was conducted in accordance with the Declaration of Helsinki (as revised in 2013). The study was approved by the Human Research Ethics Committee (Reg: LRR 205/17) of St Vincent's Hospital and individual consent for this retrospective analysis was waived.

Informed consent was not applicable or possible as this was a retrospective analysis of de-identified data with no impact on patients. A prospective database and medical records from patients who underwent surgical resection for NSCLC at St Vincent's Private and Public Hospitals in Melbourne, Australia, between January 2002 and August 2019 were retrospectively analyzed. Data obtained from hospital medical records included basic demographics, smoking status, statin use, steroid use and preoperative neutrophil:lymphocyte ratios (NLR), while tumor specific data such as pleural involvement, metastatic status and histopathology was sourced from a prospectively updated surgical database. Patients with positive resection margins, nodal involvement, metastases, or invasive chest wall disease were excluded. Patients who underwent chemotherapy were also excluded to prevent obscuration of the natural history of the disease. Patients taking corticosteroids or experiencing infections at the time of pre-operative blood sampling were not included to ensure inflammatory cell ratios were not confounded. Tumors were pathologically staged using the TNM ( $8^{\text {th }}$ revision) classification for lung cancer (9).

Follow up was until death or censorship at last known patient contact with any hospital, pathology or radiology service or medical practitioner. The outcome of interest, the first site and date of confirmed disease relapse, was 
recorded prospectively in a surgical database. The highest certainty investigation available determined the date of relapse, with pathological confirmation outranking radiological conclusion and clinical suspicion. The clinical variables chosen to represent tumor biology (differentiation on light microscopy or immunohistochemistry), tumor extent (tumor diameter and pleural invasion) and host response (NLR) were entered into a model along with other possible tumor extent factors (T-stage, $8^{\text {th }}$ revision), host factors (age, sex, smoking status) and external/system factors (insurance status, statin usage, surgical approach). A tumor was designated as 'Assignable' if it was assignable to either adenocarcinoma or squamous cell carcinoma based on morphologic features under light microscopy and/ or evidence of differentiation by immunohistochemistry according to the 2015 World Health Organization Classification of Lung Tumors (10). Otherwise, the tumor was designated as 'Unassignable', constituting a group of less differentiated histologies including neuroendocrine, pleomorphic, large cell, adenosquamous, embryonal, and mixes thereof.

\section{Statistical analysis}

NLR and tumor diameter was analyzed as both continuous and dichotomized data. Receiver operator characteristic analysis was performed to obtain the optimum cut-off values for NLR and tumor diameter. Predicted 5-year disease-free survival (DFS) was calculated by multivariate Cox regression for a series of demographic, biochemical and tumor covariates to determine effect on DFS. Pearson's $\mathrm{X}^{2}$ was used to evaluate any difference in distribution of risk factors between stage sub-groups. Hazard ratios (HR) and $95 \%$ confidence intervals (CI) were determined by Cox regression models. RStudio version 1.1.463 for Mac OSX was used to perform all comparisons of means, medians, receiver operating characteristic analyses, Kaplan-Meier estimates and Cox regression modelling.

\section{Results}

The data from 669 patients who underwent surgery for NCLC between 2002 and 2019 was analyzed. After excluding patients who had incomplete resection, positive nodal status, perioperative corticosteroid use, concomitant infection or those that received chemotherapy, a total of 394 patients remained to be included in the final analysis (355 patients in Stage I and 39 patients in Stage $\geq$ II). The range of follow-up for censored patients was 1-170 months with a median of 28 months.

The clinical and pathological characteristics of the patients are listed in Table 1. All data was available for baseline characteristics, risk factor and outcome assessment. Average patient age was 70 years (range, 20-89 years), $55 \%$ of the sample being male. Seventy-four patients $(19 \%)$ had never smoked, with the remainder being current or ex-smokers. Histopathology was represented by adenocarcinoma, squamous cell or 'unassignable' in $73 \%$, $20 \%$ and $7 \%$ of cases, respectively. Interestingly, there were no squamous cell tumors in the Stage $\geq$ II patients. Median tumor diameter was 20 and $58 \mathrm{~mm}$; and mean NLR was 2.6 and 3.2 for Stage I and Stage $\geq$ II groups, respectively.

After multivariate analysis with Cox regression, four variables were confirmed as independent predictive factors for disease recurrence (Figure 1) in a maximum efficiency model. These were NLR group $\geq 3.5$ (HR, 1.9; 95\% CI, 1.1-3.5), VPI (HR, 2.2; 95\% CI, 1.3-3.8), histology unassignable to either adenocarcinoma or squamous cell (HR, 2.6; 95\% CI, 1.2-5.5) and tumor diameter $>33 \mathrm{~mm}$ (HR, 3.9; 95\% CI, 2.3-6.7).

For Stage I tumors, $17 \%$ of patients had an NLR $\geq 3.5$, compared to $28 \%$ of patients with tumors of Stage $\geq$ II. Adenocarcinoma or squamous cell carcinoma was the morphologically and/or immunohistochemically distinct malignant histology in $93 \%$ and $90 \%$ of Stage I and Stage $\geq$ II tumors, respectively. A specific breakdown of unassignable histologies is detailed in Table 2. Only 39 (11\%) of Stage I patients, and $38(30 \%)$ of T2a patients had tumors $>33 \mathrm{~mm}$ in size. VPI was the only characteristic which significantly varied between stage groups, being identified in $26 \%$ and $51 \%$ of Stage I and Stage $\geq$ II patients, respectively $(\mathrm{P}<0.01)$.

Odds ratios from the four identified independently predictive risk factors were translated into simple scores, the value of which were determined by the approximate magnitude of effect of each risk factor. The magnitude of relative risk of recurrence from pleural involvement and NLR group was weaker compared to unassignable histology and diameter $>33 \mathrm{~mm}$, so the former groups were given scores of 0.5 and the latter, scores of 1 .

A matrix was formed from the 16 possible combinations of the four risk factor scores (Table 3). This was abbreviated to PUNT, an acronym representing the four unfavorable risk characteristics: pleural involvement, unassignable histology, NLR $\geq 3.5$ and tumor size $>33 \mathrm{~mm}$. The relapse rates for PUNT scores diverged at a score of 1.5 using Receiver Operator Characteristics, the threshold score 
Table 1 Baseline clinicopathological characteristics and distribution of risk factors between stage groupings

\begin{tabular}{|c|c|c|c|c|c|c|c|}
\hline $\begin{array}{l}\text { Variable } \\
\text { Age (years), mean }\end{array}$ & \multicolumn{2}{|c|}{ Stage I $(n=355)$} & \multicolumn{2}{|c|}{ Stage $\geq$ II $(n=39)$} & $\begin{array}{c}\text { Total }(n=394) \\
69.7\end{array}$ & $\mathrm{P}^{\mathrm{a}}$ & $\mathrm{P}\left(\chi^{2}\right)^{\mathrm{b}}$ \\
\hline Gender, n/\% & & & & & & 0.05 & \\
\hline Male & 188 & $53 \%$ & 27 & $69 \%$ & $55 \%$ & & \\
\hline Female & 167 & $47 \%$ & 12 & $31 \%$ & $45 \%$ & & \\
\hline Current or ex & 284 & $80 \%$ & 36 & $92 \%$ & $81 \%$ & & \\
\hline Never & 71 & $20 \%$ & 3 & $8 \%$ & $19 \%$ & & \\
\hline Diameter $(\mathrm{mm})$, median & \multicolumn{2}{|c|}{20} & \multicolumn{2}{|c|}{58} & 20 & \multicolumn{2}{|r|}{ N/A } \\
\hline NLR, mean & \multicolumn{2}{|c|}{2.62} & \multicolumn{2}{|c|}{3.20} & 2.68 & 0.08 & \\
\hline $1 b$ & \multicolumn{2}{|c|}{108} & & & $27 \%$ & & \\
\hline $1 c$ & \multicolumn{2}{|c|}{66} & & & $17 \%$ & & \\
\hline $2 a$ & \multicolumn{2}{|c|}{125} & & & $32 \%$ & & \\
\hline $2 b$ & & & \multicolumn{2}{|c|}{12} & $3 \%$ & & \\
\hline 3 & & & \multicolumn{2}{|c|}{14} & $4 \%$ & & \\
\hline 4 & & & \multicolumn{2}{|c|}{13} & $3 \%$ & & \\
\hline NLR group, n/\% & & & & & & & 0.10 \\
\hline Low (<3.5) & 293 & $83 \%$ & 28 & $72 \%$ & $81 \%$ & & \\
\hline Assignable $^{c}$ & 331 & $93 \%$ & 35 & $90 \%$ & $93 \%$ & & \\
\hline Unassignable & 24 & $7 \%$ & 4 & $10 \%$ & $7 \%$ & & \\
\hline Tumor size, $\mathrm{n} / \%$ & & & & & & & $\mathrm{~N} / \mathrm{A}$ \\
\hline$\leq 33 \mathrm{~mm}$ & 316 & $89 \%$ & 0 & $0 \%$ & $80 \%$ & & \\
\hline$>33 \mathrm{~mm}$ & 39 & $11 \%$ & 39 & $100 \%$ & $20 \%$ & & \\
\hline
\end{tabular}

${ }^{\mathrm{a}}, \mathrm{P}$ value calculated by student's $t$-test; ${ }^{\mathrm{b}}, \mathrm{P}$ value calculated by Chi-square test; ${ }^{\mathrm{c}}$, assignable to either squamous cell carcinoma or adenocarcinoma. N/A, not applicable; NLR, neutrophil:lymphocyte ratio; VPI, visceral pleural invasion.

assigned to the high risk of recurrence group. Kaplan Meier curves for probability of disease recurrence based on PUNT risk group were generated, revealing a $5 \mathrm{yr}$ DFS for the lowrisk and high-risk groups of $86 \%$ and $30 \%$, respectively
$(\mathrm{P}<0.001)$ (Figure 2). Thirty-one patients $(9 \%)$ in the Stage I group and twenty-seven patients $(69 \%)$ in the Stage $\geq$ II group scored $\geq 1.5$ (high-risk for recurrence) according to the PUNT model. 


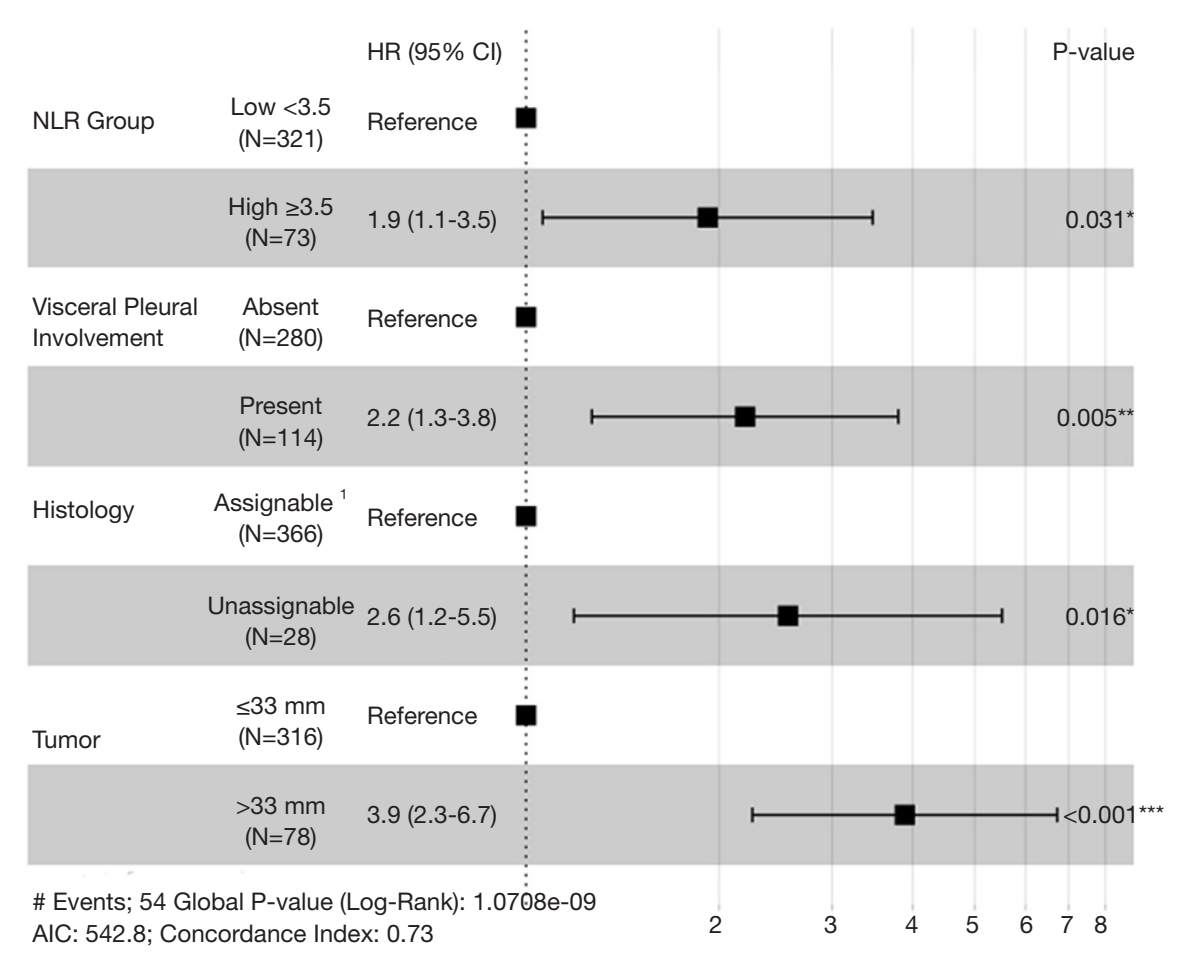

Figure 1 Forest plot of independent risk factors for disease recurrence identified by multivariate analysis. 1, adenocarcinoma or squamous cell tumors. *, $\mathrm{P}<0.05 ;{ }^{* *}, \mathrm{P}<0.01 ;{ }^{* * *}, \mathrm{P}<0.001$.

Table 2 Distribution of unassignable histology per stage groupings

\begin{tabular}{lcc}
\hline Variable & Stage I $(\mathrm{n}=24)$ & Stage $\geq \mathrm{II}(\mathrm{n}=4)$ \\
\hline Large cell & 2 & 0 \\
Large cell/neuroendocrine & 6 & 1 \\
Adeno/neuroendocrine & 3 & 0 \\
Adenosquamous & 1 & 1 \\
Squamous/neuroendocrine & 1 & 0 \\
Embryonal & 0 & 1 \\
Pleomorphic & 11 & 1 \\
\hline
\end{tabular}

\section{Discussion}

The present study identified four risk factors that independently predicted disease recurrence risk in a cohort of completely resected, node-negative, NSCLC patients. These represent one host factor, two tumor extent factors and one tumor grade factor that are easily collected or measured in routine practice.

There has been an extensive array of investigations into the prognostic implication of inflammatory cell ratios in a veritable pantheon of diseases, from acute coronary syndrome and stroke to a range of solid malignancies (11-13). Of the ratios, the most well studied and productive of consistent results is the NLR. A high NLR, although a peripheral blood measurement, is thought to be reflective of the imbalance in neutrophil driven pro-inflammatory processes over immuno-regulatory lymphocyte function that constitutes the milieu of the tumor microenvironment, contributing to tumor growth, invasion, metastasis and subsequently recurrence.

Despite the lack of clear consensus on exact cutoff values, studies have consistently linked a higher NLR status with worse overall and DFS. A 2015 metanalysis found increased disease recurrence rates with higher NLRs involving all stages of resected NSCLC (14). Studies confined to early stage NSCLC, have similarly confirmed greater recurrence rates in patients with a high NLR on multivariate analysis. In 343 completely resected NSCLC stage I-IIA patients, preoperative NLR >2.5 predicted worse DFS (HR, 2.141; 95\% CI, 1.306-3.515, P=0.003) (15). Even in stage IA resected NSCLC, an NLR $>3.5$ was associated with worse 
Table 3 PUNT scoring matrix for predicting 5-year disease-free survival

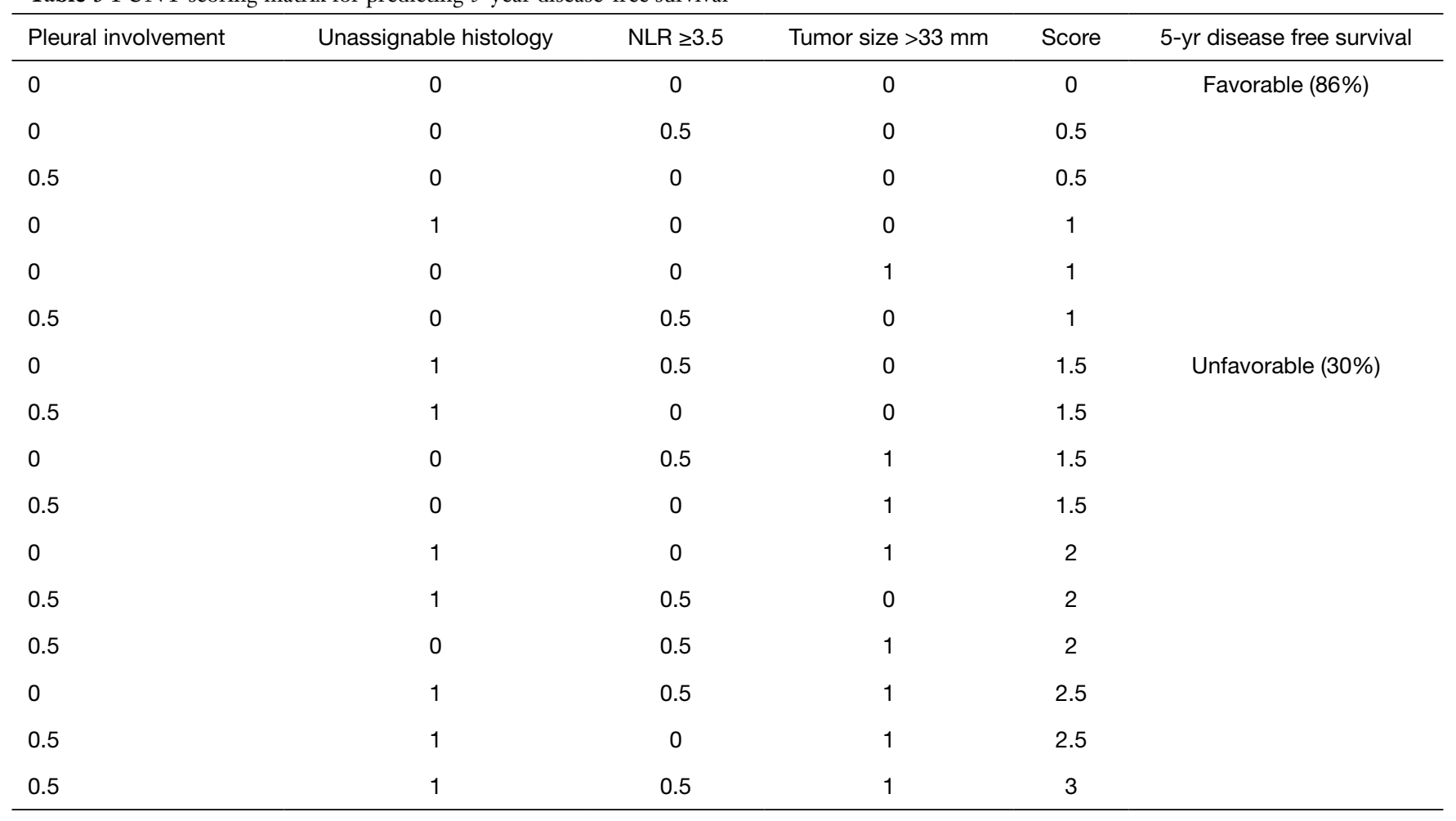

NLR, neutrophil:lymphocyte ratio.

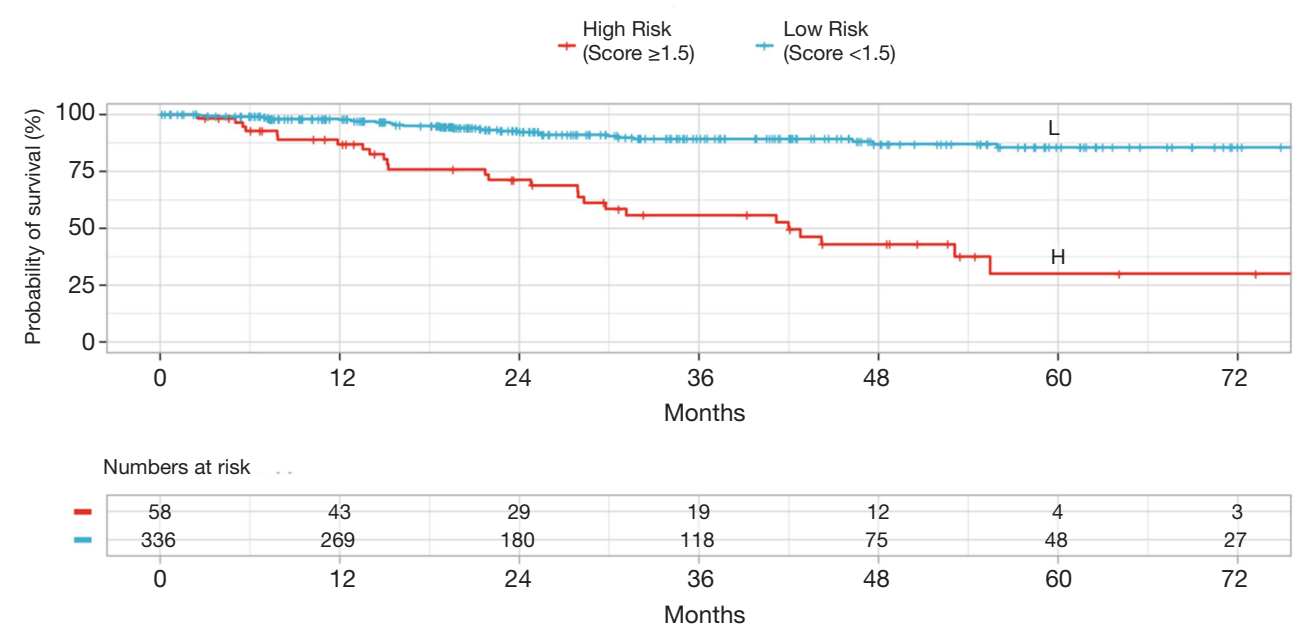

Figure 2 Kaplan-Meier curves for 5 -year DFS by risk status on the PUNT predictive model. 5 Yr (low risk) DFS =86\% vs. 5 Yr (high risk) DFS $=30 \%, \mathrm{P}<0.001$. DFS, disease-free survival.

recurrence free survival (HR, 2.03, 95\% CI, 1.17-3.79; $\mathrm{P}=0.01)$ (16). Discrepancy in NLR cutoffs could be explained by heterogeneity of sample populations and adjuvant therapies, population demographics, sample timing and statistical method. Allowing for different cutoffs, our finding of an NLR $\geq 3.5$ predicting risk of recurrence at a HR of 1.9 (95\% CI, 1.1-3.5, $\mathrm{P}=0.031)$ falls in line with contemporary findings. 
It is well established that DFS and OS (9) reduces with increasing tumor size. A single size threshold at which survival reduces significantly or at which AC should be considered has not been determined. This is especially true for resected, node-negative tumors of size 31 to $40 \mathrm{~mm}$. A 2016 study analyzing 25,267 patients from the National Cancer Data Base determined that AC improved OS in all T2N0M0 tumors (including under $40 \mathrm{~mm}$ ) regardless of size (17). Alternatively, a sub-group analysis of the CALGB 9633 trial demonstrated survival benefit of AC only in tumors $\geq 40 \mathrm{~mm}$ in size (18).

While tumor size alone does have an impact on survival and response to chemotherapy, the presence of other highrisk features significantly adds to the prognostic algorithm. Recently, a comprehensive analysis of 50,814 patients with node-negative NSCLC from the National Cancer Database assessed the effect of AC on survival based on tumor size categories and high-risk pathological factors (VPI, lymphovascular invasion, sub-lobar resection and high-grade histological findings) (19). The authors found that AC conferred survival benefit in tumors $31-40 \mathrm{~mm}$ in those who underwent sub-lobar resection (HR, 0.72; 95\% CI, 0.56-0.93; $\mathrm{P}=0.004$ ) and for tumors $41-50 \mathrm{~mm}$ in cases possessing at least one high-risk feature (HR, 0.67; 95\% CI, 0.56-0.80; $\mathrm{P}=0.02$ ). Once the $50 \mathrm{~mm}$ tumor threshold was breached however, high risk features no longer were required to obtain a survival advantage with chemotherapy (HR, 0.75; 95\% CI, 0.61-0.91; $\mathrm{P}=0.004$ ).

The complex interplay between size and high-risk features and their effect on survival is reflected in the guidelines. The European Society for Medical Oncology clinical practical guidelines do not endorse chemotherapy in T2aNOM0 resected NSCLC (20). The American Society of Clinical Oncology does not support routine use of AC in Stage IB, instead recommending an individualized riskbenefit evaluation (21). The 2019 National Comprehensive Cancer Network guidelines advocate for either observation or AC if certain high-risk features are present (poorly differentiated tumors, vascular invasion, wedge resection, tumors $>40 \mathrm{~mm}$, VPI and unknown lymph node status) (6).

Given the degree of prognostic uncertainty that arises from the stage IB sub-group, it is unsurprising that that the present study identified a higher disease recurrence risk with tumors $34 \mathrm{~mm}$ or above, a cut off falling roughly in the center of the stage IB size range. In addition, tumor size exhibited the greatest effect (HR, 3.9; 95\% CI, 2.3-6.7; $\mathrm{P}<0.001$ ) on disease recurrence out of the four risk factors identified in this study, which confirms the importance of tumor size generally as a prognostic marker.

For histological risk factor analysis, malignancies with a differentiated histology such as squamous cell and adenocarcinoma were grouped under the 'assignable' title and all other NSCLC types were designated as 'unassignable'. These rarer, poorly differentiated tumors, such as neuroendocrine, pleomorphic (giant cell or sarcomalike mesenchymal), muco-epidermoid, adenosquamous or large cell are recognized to have a much poorer outcome than the more common adenocarcinoma or squamous tumors (22-24). Given this knowledge, it is understandable that this was the second most powerful predictor of recurrence on multivariate analysis in the present study with the unassignable group predicting recurrence at a HR of 2.6 (95\% CI, 1.2-5.5, $\mathrm{P}=0.016$ ).

Analysis was not made on histology grade as there was insufficient data for all histology types to provide an accurate comparison. This is an area however that has been rigorously explored and a common understanding of the importance of histological grade in prognostic endeavors has fermented. Broadly speaking, the degree of tumor cell differentiation is positively associated with OS and DFS regardless of NSCLC subtype (25).

No validated system exists for grading squamous cell lung carcinoma, but for adenocarcinoma specifically, the 2011 International Association for the Study of Lung Cancer/American Thoracic Society/European Respiratory Society (26) and the 2015 World Health Organization classification system (10) distinguished the majority of adenocarcinoma tumors, which exhibit a mixed histological pattern, into five main subtypes according to the predominant histological pattern. These can be further grouped into three divisions based on varying survival outcomes with lepidic predominant; acinar/papillary predominant; and micropapillary/solid predominant associated with good, intermediate, and poorer outcomes, respectively (27-29). In 2015, Tsao et al. (30) analyzed 575 cases of adenocarcinoma from the LACE-Bio data set for the impact of subtype on survival and benefit from AC. Compared to the acinar/papillary subtype, the micropapillary/solid predominant pattern not only had a significantly worse DFS (HR, 1.52; 95\% CI, 1.09 to 2.11; $\mathrm{P}=0.01)$ and specific disease-free-survival (SDFS) (HR, 1.58; $95 \% \mathrm{CI}, 1.11$ to $2.23 ; \mathrm{P}=0.01$ ) but was also associated with a significant DFS (HR, $0.65 ; 95 \%$ CI, 0.48 to $0.88 ; \mathrm{P}<0.01$ ) and SDFS (HR, $0.63 ; 95 \%$ CI, 0.46 to $0.87 ; \mathrm{P}<0.01$ ) benefit from AC.

One challenge which remains in the pathological 
Table 4 Distribution of PUNT risk category by TNM stage. This is represented by absolute numbers, n, and percentage (\%), for each TNM stage

\begin{tabular}{lcccccccc}
\hline Score (risk) & 5 -yr DFS & Stage I, $\mathrm{n}=355$ & Stage IA, $\mathrm{n}=230$ & Stage IB, $\mathrm{n}=125$ & Stage $\geq$ II, $\mathrm{n}=39$ & Stage II, $\mathrm{n}=26$ & Stage IIIA, $\mathrm{n}=13$ & Total, $\mathrm{n}=394$ \\
\hline$<1.5$ (low) & $86 \%$ & $324(91 \%)$ & $227(99 \%)$ & $97(78 \%)$ & $12(31 \%)$ & $8(30 \%)$ & $4(30 \%)$ & $336(85 \%)$ \\
$\geq 1.5$ (high) & $30 \%$ & $31(9 \%)$ & $3(1 \%)$ & $28(22 \%)$ & $27(69 \%)$ & $18(70 \%)$ & $9(70 \%)$ & $58(15 \%)$ \\
\hline
\end{tabular}

diagnosis of adenocarcinoma subgroups is the achievement of consistent reporting within and between institutions. Shortly after the IASLC/ATS/ERS was published, validation studies emerged testing the reliability of pathological reporting. One study demonstrated interobserver reliability to be moderate to substantial $(\mathrm{k}=0.44-0.72)$ (31), with consistency being affected by training, specific sub type being analyzed and number of slides examined. Another study revealed good inter-observer reliability when typical slides were tested $(\mathrm{k}=0.77 \pm 0.07)$ but worse when slides being analyzed were assessed as difficult $(k=0.38 \pm 0.14)$, with the greatest overlap in identification being between papillary and micropapillary predominance (32). Despite the lingering limitations in pathological identification of histological grade mentioned above, NSCLC subtype remains an important and readily accessible predictive factor and would likely be a useful addition to the prognostic algorithm in the future, particularly for adenocarcinoma.

Our finding that visceral pleural invasion predicted recurrence (HR, 2.2; $95 \% \mathrm{CI}, 1.3-3.8 ; \mathrm{P}=0.005)$ is consistent with most reported literature. An analysis of 16,315 patients with resected stage I-II NSCLC demonstrated that VPI was associated with worse cancer specific survival (HR, 1.38; 95\% CI, 1.29-1.47) (33). In another study looking specifically at a subset of IB patients, some of whom received AC, after adjustment, VPI was associated with a significantly poorer DFS (HR, 7.53; 95\% CI, 1.66-34.06; $\mathrm{P}=0.009)$ and OS (HR, 8.75; 95\% CI, 1.09-70.39; $\mathrm{P}=0.041$ ) (34). The presence of VPI has been repeatedly demonstrated to prognostically upstage patients $(35,36)$.

By compounding the effects of readily available clinical and pathological markers of tumor and host response, the PUNT prognostic model turned out to be a simple yet powerful method of distinguishing sub-groups in each stage of resectable NSCLC that have a discordantly high or low risk of recurrence (Table 4). According to the model, 31 of the $355(9 \%)$ patients in the Stage I group were designated as high risk, giving them a 5 -yr DFS of approximately $30 \%$, far below the expected 5 -yr DFS of $72-96 \%$ (37) for Stage I patients. The overwhelming majority of the high- risk patients in the Stage I group (28/31) were identified in the IB sub-group. Of all the stage IB patients in this study ( $\mathrm{n}=125), 28$ patients $(22 \%)$ turned out to be high risk, which suggests that this model could be especially useful in triaging risk profile, consideration of $\mathrm{AC}$ and surveillance frequency in this controversial population. Conversely, the Stage $\geq$ II group, which would usually have predicted 5-yr DFS of 40-65\% (37), contained twelve out of 39 patients with PUNT scores designating them as low risk and attributing them an expected $5 \mathrm{yr}$ DFS of $86 \%$. These twelve low-risk patients, by nature of their stage, had larger tumors, but lacked any of the three other high-risk features in the model. The proportion of low risk patientsapproximately $30 \%$ - did not differ between Stage II or Stage IIIA. These findings indicate that $31 \%$ of the Stage $\geq$ II group had such a favorable prognosis as to potentially tip the risk-benefit balance toward omission of AC.

This study was limited by the retrospective nature of the analysis and by the relatively small numbers. Data for some currently experimental and some other more established risk factors which may be relevant in prognostication of NSCLC were not available in the present study and would be a useful target of investigation in the future. Despite not including various previously validated risk factors, the four readily accessible and reliable risk factors identified in the study when combined together as part of a risk stratification model yielded a vast divergence in survival curves associated with a high degree of statistical significance. As this predictive model has been developed from a single institution in an advanced health and economic setting, it would be necessary to validate this model in similar and different settings and populations.

It is also important to recognize that despite currently being limited to clinical trials of small sample sizes and reliance on surrogate markers of survival rather than time-to-event end points, there is evolving evidence for immunotherapy in both neoadjuvant and adjuvant settings in early stage, NSCLC (38). The current study's assumptions are formed juxtaposed against the backdrop of contemporary best practice, and this is likely also to evolve based on emerging evidence and changing 
guidelines. Therefore, validation of the PUNT tool in various populations, including those being considered for immunotherapy would be prudent.

\section{Conclusions}

Based on four readily accessible and reproducible clinical and pathological factors independently predicting disease recurrence, the present study yielded a recurrence-risk scoring system (PUNT) that identified sub-groups of patients who were at significantly higher or lower risk of recurrence than expected for their TNM stage. If validated, this tool could facilitate a more targeted algorithm for adjuvant systemic therapy decisions in completely resected, node-negative NSCLC patients.

\section{Acknowledgments}

The authors would like to acknowledge the staff at health information systems at St Vincent's Public and Private Hospitals, Melbourne, for assistance in file procurement. The authors would also like to thank Jane Mack, cardiothoracic research coordinator and her team at the cardiothoracic department at St Vincent's Hospital Melbourne for administrative assistance and advice.

Funding: None.

\section{Footnote}

Reporting Checklist: The authors have completed the TRIPOD reporting checklist. Available at http://dx.doi. org/10.21037/jtd-20-2434

Data Sharing Statement: Available at http://dx.doi. org/10.21037/jtd-20-2434

Conflicts of Interest: All authors have completed the ICMJE uniform disclosure form (available at http://dx.doi. org/10.21037/jtd-20-2434). The authors have no conflicts of interest to declare.

Ethical Statement: The authors are accountable for all aspects of the work in ensuring that questions related to the accuracy or integrity of any part of the work are appropriately investigated and resolved. The study was conducted in accordance with the Declaration of Helsinki (as revised in 2013). The study was approved by the Human Research Ethics Committee [Reg: LRR 205/17] of St Vincent's Hospital and individual consent for this retrospective analysis was waived. Informed consent was not applicable or possible as this was a retrospective analysis of de-identified data with no impact on patients.

Open Access Statement: This is an Open Access article distributed in accordance with the Creative Commons Attribution-NonCommercial-NoDerivs 4.0 International License (CC BY-NC-ND 4.0), which permits the noncommercial replication and distribution of the article with the strict proviso that no changes or edits are made and the original work is properly cited (including links to both the formal publication through the relevant DOI and the license). See: https://creativecommons.org/licenses/by-nc-nd/4.0/.

\section{References}

1. Kelsey CR, Marks LB, Hollis D, et al. Local recurrence after surgery for early stage lung cancer: an 11-year experience with 975 patients. Cancer 2009;115:5218-27.

2. Lou F, Huang J, Sima CS, et al. Patterns of recurrence and second primary lung cancer in early-stage lung cancer survivors followed with routine computed tomography surveillance. J Thorac Cardiovasc Surg 2013;145:75-81.

3. Burdett S, Pignon JP, Tierney J, et al. Adjuvant chemotherapy for resected early-stage non-small cell lung cancer. Cochrane Database Syst Rev 2015;(3):CD011430.

4. Pignon JP, Tribodet H, Scagliotti GV, et al. Lung adjuvant cisplatin evaluation: a pooled analysis by the LACE Collaborative Group. J Clin Oncol 2008;26:3552-9.

5. Arriagada R, Auperin A, Burdett S, et al. Adjuvant chemotherapy, with or without postoperative radiotherapy, in operable non-small cell lung cancer: two meta-analyses of individual patient data. Lancet 2010;375:1267-77.

6. National Comprehensive Cancer Network. Non-small cell lung cancer. (Version 7.2019). Available online: https:// www.nccn.org/store/login/login.aspx?ReturnURL=https:// www.nccn.org/professionals/physician_gls/pdf/nscl.pdf. Accessed November 5, 2019.

7. Uramoto H, Tanaka F. Prediction of recurrence after complete resection in patients with NSCLC. Anticancer Res 2012;32:3953-60.

8. Collins GS, Reitsma JB, Altman DG, et al. Transparent reporting of a multivariable prediction model for individual prognosis or diagnosis (TRIPOD): The TRIPOD statement. Ann Intern Med 2015;162:55-63.

9. Goldstraw P, Chansky K, Crowley J, et al. The IASLC lung cancer staging project: proposals for revision of the 
TNM stage groupings in the forthcoming (eighth) edition of the TNM classification for lung cancer. J Thorac Oncol 2016;11:39-51.

10. Travis WD, Brambilla E, Nicholson AG, et al. The 2015 World Health Organization Classification of Lung Tumors: Impact of Genetic, Clinical and Radiologic Advances Since the 2004 Classification. J Thorac Oncol 2015;10:1243-60.

11. Zhang S, Diao J, Qi C, et al. Predictive value of neutrophil to lymphocyte ratio in patients with acute ST segment elevation myocardial infarction after percutaneous coronary intervention: a meta-analysis. BMC Cardiovasc Disord 2018;18:75.

12. Song SY, Zhao XX, Rajah G, et al. Clinical significance of baseline neutrophil-to-lymphocyte ratio in patients with ischemic stroke or hemorrhagic stroke: an updated metaanalysis. Front Neurol 2019;10:1032.

13. Templeton AJ, McNamara MG, Šeruga B, et al. Prognostic role of neutrophil-to-lymphocyte ratio in solid tumors: a systematic review and meta-analysis. J Natl Cancer Inst 2014;106:dju124.

14. Gu XB, Tian T, Tian XJ, et al. Prognostic significance of neutrophil-to-lymphocyte ratio in non-small cell lung cancer: a meta-analysis. Sci Rep 2015;5:12493.

15. Takahashi Y, Horio H, Hato T, et al. Prognostic significance of preoperative neutrophil-lymphocyte ratios in patients with stage I non-small cell lung cancer after complete resection. Ann Surg Oncol 2015;22:S1324-31.

16. Mizuguchi S, Izumi N, Tsukioka T, et al. Neutrophillymphocyte ratio predicts recurrence in patients with resected stage1 non-small cell lung cancer. J Cardiothoracic Surg 2018;13:1-7.

17. Morgensztern D, Du L, Waqar SN, et al. Adjuvant chemotherapy for patients with T2N0M0 NSCLC. J Thorac Oncol 2016;11:1729-35.

18. Strauss GM, Herndon II JE, Maddaus MA, et al. Adjuvant paclitaxel plus carboplatin compared with observation in stage IB non-small-cell lung cancer: CALGB 9633 with the Cancer and Leukemia Group B, Radiation Therapy Oncology Group, and North Central Cancer Treatment Group Study Groups. J Clin Oncol 2008;26:5043-51.

19. Pathak R, Goldberg SB, Canavan M, et al. Association of Survival With Adjuvant Chemotherapy Among Patients With Early-Stage Non-Small Cell Lung Cancer With vs. Without High-Risk Clinicopathologic Features. JAMA Oncol 2020;6:1-10.

20. Postmus PE, Kerr KM, Oudkerk M, et al. Early and locally advanced non-small-cell lung cancer (NSCLC):
ESMO clinical practice guidelines for diagnosis, treatment and follow-up. Ann Oncol 2017;28:1-21.

21. Kris MG, Gaspar LE, Chaft JE, et al. Adjuvant Systemic Therapy and Adjuvant Radiation Therapy for Stage I to IIIA Completely Resected Non-Small-Cell Lung Cancers: American Society of Clinical Oncology/Cancer Care Ontario Clinical Practice Guideline Update. J Clin Oncol 2017;35:2960-74.

22. Han K, Yang H, Fan L, et al. Outcomes of patients with large cell neuroendocrine carcinoma of the lung after complete resection. Transl Cancer Res 2017;6:483-92.

23. Cooke DT, Nguyen DV, Yang Y, et al. Survival comparison of adenosquamous, squamous cell, and adenocarcinoma of the lung after lobectomy. Ann Thorac Surg 2010;90:943-8.

24. Mochizuki T, Ishii G, Nagai K, et al. Pleomorphic carcinoma of the lung: clinicopathologic characteristics of 70 cases. Am J Surg Pathol 2008;32:1727-35.

25. Yasukawa M, Sawabata N, Kawaguchi T, et al. Histological grade: analysis of prognosis of non-small cell lung cancer after complete resection. In Vivo 2018;32:1505-12.

26. Travis WD, Brambilla E, Noguchi M, et al. International association for the study of lung cancer/American Thoracic Society/European Respiratory Society International multidisciplinary classification of lung adenocarcinoma. J Thorac Oncol 2011;6:244-85.

27. Russell PA, Wainer Z, Wright GM, et al. Does lung adenocarcinoma subtype predict patient survival? A clinicopathologic study based on the new International Association for the Study of Lung Cancer/American Thoracic Society/European Respiratory Society International Multidisciplinary Lung Adenocarcinoma Classification. J Thorac Oncol 2011;6:1496-504.

28. Warth A, Muley T, Meister M, et al. The Novel Histologic International Association for the Study of Lung Cancer/ American Thoracic Society/European Respiratory Society Classification System of Lung Adenocarcinoma is a stage-independent predictor of survival. J Clin Oncol 2012;30:1438-46.

29. Yoshizawa A, Motoi N, Riely GJ, et al. Impact of proposed IASLC/ATS/ERS classification of lung adenocarcinoma: prognostic subgroups and implications for further revision of staging based on analysis of 514 stage I cases. Mod Pathol 2011;24:653-64.

30. Tsao MS, Marguet S, Le Teuff G, et al. Subtype classification of lung adenocarcinoma predicts benefit from adjuvant chemotherapy in patients undergoing complete resection. J Clin Oncol 2015;33:3439-46.

31. Warth A, Stenzinger A, von Brunneck AC, et al. 
Interobserver variability in the application of the novel IASLC/ATS/ERS classification for pulmonary adenocarcinomas. Eur Respir J 2012;40:1221-7.

32. Thunnissen E, Beasley MB, Borczuk AC, et al. Reproducibility of histopathological subtypes and invasion in pulmonary adenocarcinoma: An international interobserver study. Mod Pathol 2012;25:1574-83.

33. Lakha S, Gomez JE, Flores RM, et al. Prognostic significance of visceral pleural involvement in early-stage lung cancer. Chest 2014;146:1619-26.

34. Park HJ, Park HS, Cha YJ, et al. Efficacy of adjuvant chemotherapy for completely resected stage IB nonsmall cell lung cancer: a retrospective study. J Thorac Dis 2018;10:2279-87.

35. Fibla JJ, Cassivi SD, Brunelli A, et al. Re-evaluation of the prognostic value of visceral pleura invasion in stage

Cite this article as: Krivitsky TA, Wright GM, Al Zaidi M. A predictive model for identifying candidates for adjuvant chemotherapy based on recurrence risk profile of resected, node-negative (N0) non-small cell lung cancer. J Thorac Dis 2021;13(1):149-159. doi: 10.21037/jtd-20-2434
IB non-small cell lung cancer using the prospective multicenter ACOSOG Z0030 trial data set. Lung Cancer 2012;78:259-62.

36. Zhang T, Zhang JT, Li WF, et al. Visceral pleural invasion in T1 tumors $(\leq 3 \mathrm{~cm})$, particularly T1a, in the eighth tumor-node-metastasis classification system for non-small cell lung cancer: a population-based study. J Thorac Dis 2019;11:2754-62.

37. Yun JK, Lee GD, Kim HR, et al. Validation of the 8th edition of the TNM staging system in 3,950 patients with surgically resected non-small cell lung cancer. J Thorac Dis 2019;11:2955-64.

38. Uprety D, Mandrekar SJ, Wigle D, et al. Neoadjuvant Immunotherapy for NSCLC: Current Concepts and Future Approaches. J Thorac Oncol 2020;15:1281-97. 